\title{
Automatic techniques and machine learning to improve stratigraphic interpretation
}

Raisa Carvalho and Maria González, Emerson Automation Solutions.

Copyright 2021, SBGf - Sociedade Brasileira de Geofísica

This paper was prepared for presentation during the $17^{\text {th }}$ International Congress of the Brazilian Geophysical Society held in Rio de Janeiro, Brazil, 16-19 August 2021.

Contents of this paper were reviewed by the Technical Committee of the $17^{\text {th }}$ International Congress of the Brazilian Geophysical Society and do not necessarily represent any position of the SBGf its officers or members. Electronic reproduction or storage of any part of this paper for commercial purposes without the written consent of the Brazilian Geophysical Society is prohibited.

\section{Abstract}

Stratigraphic interpretation workflows pursuits a better understanding of the reservoirs. Seismic data provides important information through detailed analysis of its response and extracted attributes to identify the geometry and extension of stratigraphic features and possible changes in lithology or fluid content. The challenge in this case, is to find a combination of methods and best practices that allow the interpreters to explore the seismic data in an effective manner and unveil hidden patterns from the information. Machine learning techniques applied to seismic interpretation have been successful in this regard and very useful in assisting with limitations involving big volumes of data, automation, and data classification. In this sense, the stratigraphic interpretation becomes a more automatic and reliable process.

In this work, we aimed to show how advanced visualization techniques, with a proper combination of attributes and an efficient machine learning method, as Self Growing Neural Network, can improve the stratigraphic interpretation process, generate accurate results and provide information for uncertainty analysis.

\section{Introduction}

In hydrocarbon exploration and production, the understanding of the reservoir stratigraphy is a key factor to succeed in the field development. Seismic data provides information for prospect delineation, geometric relationships between layers and changes in lithology or fluid content. Improving the way interpreters explore and handle seismic data can make the difference in the quality of their results.

Seismic attributes are very effective tools to emphasize stratigraphic features that can be easily detected, or not, in seismic amplitude. Taner (2000) defines the seismic attributes as any information obtained from seismic data, either they are logic or based in experience or previous knowledge.

Thus, for an effective stratigraphic interpretation, first we need to compute seismic attributes and combine them in a skillful way, and with the support of some visualization techniques, we try to obtain as much information as possible to identify the geometry and extension of the stratigraphic features.

After an exhaustive study of the stratigraphic information from seismic attributes, the next step is to perform a facies classification. Here, we can use machine learning techniques to extract information and hidden patterns through a variety of algorithms that look for nonlinear correlations. The concept of machine learning has been a key element for the advance in this aspect, allowing a more detailed analysis and more reliable results.

Machine learning algorithms have demonstrated to be useful to solve problems related to huge volume of data: classification, automation, transformation, and integration. Currently, the analysis of seismic patterns uses this type of technology and geostatistical methods to produce most probable facies, and probabilities volumes for each facies providing information for uncertainty analysis also.

The purpose of this work is to show a methodology for an exhaustive and automatic stratigraphic interpretation, with a non-supervised facies classification that uses SelfGrowing Neural Network (SGNN), a machine learning algorithm based in Growing Neural Gas (Fritzke 1995). As result, we have a most probable facies volume that allow us to extract automatically geobodies related to the reservoir facies and probability volumes for each facies.

\section{Method}

The following steps were adopted for an automatic stratigraphic interpretation, as shown in Figure 1:

(I) Definition of the seismic interval: this is the first step to explore the seismic data, restricting our search for information to a volume or interval that contains the features of interest.

(II) Seismic attributes calculation: seismic attributes are mathematical descriptions of the shape of a seismic trace in specific time intervals (Schroeder, 2011). They enable interpreters to highlight some features that may not be identified with the seismic amplitude.

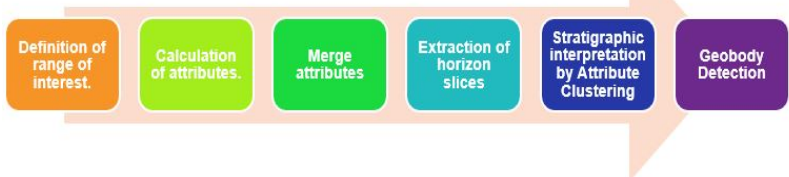

Figure 1: Flowchart with the steps followed in the methodology: (I) Definition of the interval of interest, (II) 
calculation of attributes, (III) merge of attributes, (IV) extraction of horizon slices, (V) stratigraphic interpretation by attributes clustering and (VI) geobody detection.

The following attributes were calculated and analyzed in this work:

Signal Envelope: It is independent of the phase and represents the total instantaneous energy of the seismic trace. It is used to highlight discontinuities, lithological changes, faults, channels, unconformities, and gas accumulations.

Eigen coherence: It is the ratio of the energy of the coherent component of the data to the energy of the original traces within the analysis window. The eigenstructure method analyzes a window of traces and determines which wavelet best represents the waveform variability. This wavelet is scaled to fit each input trace, providing the coherence component of the data (Chopra et al., 2007). It is very useful to understand the lateral extension of meaningful geologic features and for structural and stratigraphic interpretation.

Spectral Decomposition: It is used to highlight geologic features that have a different response in different frequency bands of the seismic trace. The seismic amplitude is decomposed in multiple frequency bands using the Fourier transform (Partyka, 1999). It is helpful in improving the resolution of seismic events, channel identification, thin bed detection, and thickness estimation of layers.

(III) Merge of attributes or color blend using addition and RGB (Red, Green, Blue) methods. The combination of attributes is frequently used to enhance the visualization of seismic data and show details and trends from different sources. Improved visualization techniques have made possible to refine stratigraphic analysis and interpretation workflows on an exploratory scale. This process of blending attribute colors can be performed with up to three attributes, simultaneously, allowing the identification of unseen or less evident features (Henderson et al., 2008; Henderson et al., 2007).

There are several techniques to color blend the attributes, but only two of them were applied in our workflow: attribute values (RGB) and addition.

The attribute value (RGB) method assigns each volume to a unique monochromatic palette (Red, Green and Blue) and the color varies from light to dark in the same palette. The data is mapped so the higher amplitudes correspond to the darkest colors (higher RGB). Then, the volumes are merge using the addition method.

In the addition method, the interpreter should select the color palette for each attribute that provides the best contrast when merged. The selection of the color palette is essential to obtain an optimized display.

(IV) Extraction of horizon slices
It's a method to explore the seismic volume to interpret the geometry of geologic bodies

This extraction can be accomplished using one horizon as reference or between two horizons. In this workflow, we extracted horizon slices in the seismic interval of interest.

(V) Stratigraphic interpretation using Attribute Clustering

Attribute clustering is a method for an automatic non supervised facies classification, using a machine learning algorithm known as Self-Growing Neural Network or Growing Neural Gas (Fritzke, 1995). This model uses a system based on neurons that behave as a gas during the training process.

The SGNN technique builds a topology referential defined dynamically and keeps the neighborhood relationships of the data. Two principles are honored: i) two neighbor elements in data space continue as neighbors in the topology space, ii) more neurons are assigned to regions with dense level of information. Thus, several neuron families are created based on the data similarity and propagated later into seismic by using a Bayesian approach (Hami-Eddine et al., 2015).

Figure 2 shows the complete workflow to create the facies volume:

- Input data: selection of seismic attributes and the interval for the classification.

- Training: the neural network is trained on the input data and identifies main data trends that can be used to classify the data.

- Classification: generate a facies volume, with related probability outputs. The trained neural network will be used during this step to estimate probabilities for each facies.

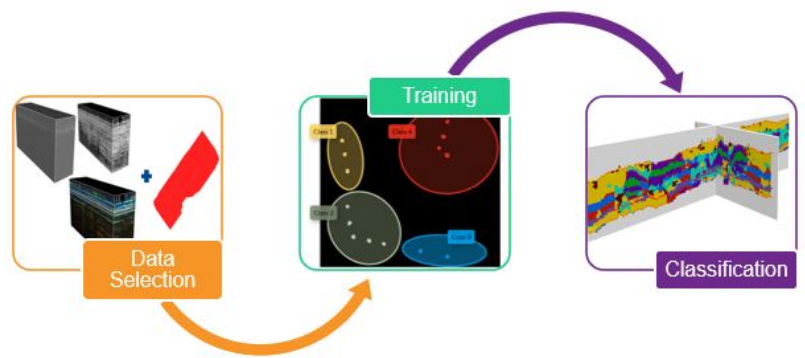

Figure 2: Steps for the facies classification by the attribute clustering workflow, which extract the seismic patterns and create most probable facies volume.

\section{(VI) Geobody Detection:}

In this step, we isolate the geobody that represents our feature of interest considering its facies value.

Results

In order to demonstrate the methodology, a dataset from Western Saskatchewan, Canada, was chosen. The targets are channels with gas accumulations from Colony Formation. 
The target channels are shown in Figure 3A, at time slice $506 \mathrm{~ms}$, extracted from the seismic amplitude. Other attributes were calculated to enhance the image of the channel system and identify the boundaries, so we could define the interval of interest for the analysis. Those seismic attributes were also input for the facies classification process.

An eigen coherence was created from the seismic amplitude (Figure 3B). This attribute highlights discontinuities on the seismic data and gives a more detailed image of the channel boundaries. Also, a spectral decomposition on the amplitude envelope was computed at $10 \mathrm{~Hz}, 37.5 \mathrm{~Hz}$ and $65 \mathrm{~Hz}$ (Figures 3C, 3D and 3E). To obtain a better visualization of the channels, we merged the attributes using the RGB and addition methods.

In Figure $3 \mathrm{~F}$ with the blending in $\mathrm{RGB}$ of $10 \mathrm{~Hz}$ component plus amplitude and eigen coherence, we noticed a better delimitation of the channel's borders, given by the coherence. Figure $3 G$ shows the merge of the three spectral decomposition volumes highlighting the brighter areas, probably related to gas accumulations. Figure $3 \mathrm{H}$ displays the color blending by addition of eigen coherence, amplitude and $10 \mathrm{~Hz}$ spectral decomposition component.

To set the interval of interest for the facies classification, we used the Waseca horizon as reference. It represents the bottom of Colony Formation. Horizon slices were created every $8 \mathrm{~ms}$, up and down from the reference horizon, to study the continuity of the channels (Figure 4). In these horizons, we could extract the merged attributes and see the variation of the channel stratigraphy. Finally, the top of the interval of interest was set at $-40 \mathrm{~ms}$ from the Waseca horizon and the bottom at $10 \mathrm{~ms}$.

The eigen coherence, spectral decomposition on the envelope at $10 \mathrm{~Hz}, 37.5 \mathrm{~Hz}$ and $65 \mathrm{~Hz}$, and the amplitude volumes were the input data for the non-supervised facies classification. Several training runs were carried out until we found an optimal number of neurons. In this case, a model with 7 classes gave the best results to represent the target channels.

Some quality controls were performed to guarantee more confidence in the results. For example, a parallel plot allows the assessment of every attribute contribution during the neuron training (Figure 5). In this case, there is a good distribution or variation of every class neuron for the different attributes, confirming that all the attributes contribute for the prediction.

During the classification stage, we use a $\mathrm{K}$ Nearest Neighbor (KNN) method, which allows the estimation of the most probable facies by defining the neuron influence area as a Gaussian function (weight $=\mathrm{e}^{- \text {distance }}$ ). Figure $6 \mathrm{~A}$ shows the most probable facies volume and the probability for each class obtained. Finally, a smoothing is performed on the results (Figure 6B), using the seismic dip and azimuth volumes. Maximum facies probability is also created to check in every sample the occurrence probability of the facies. This type of information is very useful for uncertainty analysis associated to facies modeling.

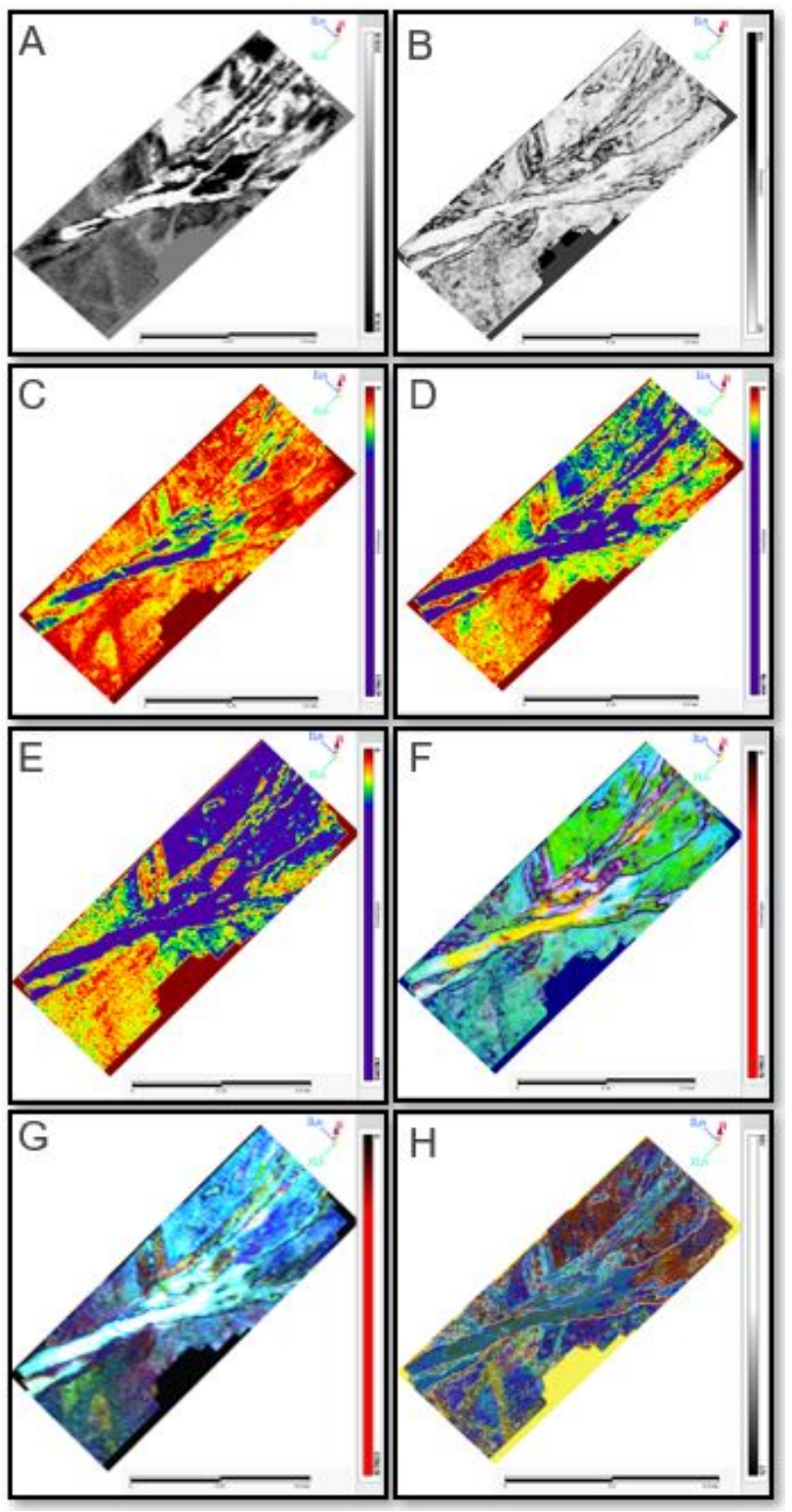

Figure 3: Time slice extracted at $506 \mathrm{~ms}$ from the following attributes: A) Seismic amplitude, B) Eigen coherence C) Spectral decomposition of envelope at $10 \mathrm{~Hz}$, D) Spectral decomposition of envelope at $37.5 \mathrm{~Hz}$, E) Spectral decomposition of envelope at $65 \mathrm{~Hz}, \quad$ F) Merge of eigen coherence, spectral decomposition at $10 \mathrm{~Hz}$ and amplitude by RGB method, G) Blending of spectral decomposition at $10 \mathrm{~Hz}, 37.5 \mathrm{~Hz}$ and $65 \mathrm{~Hz}$, by RGB method, H) Merge of eigen coherence, spectral decomposition at $10 \mathrm{~Hz}$ and amplitude, by the addition method. 


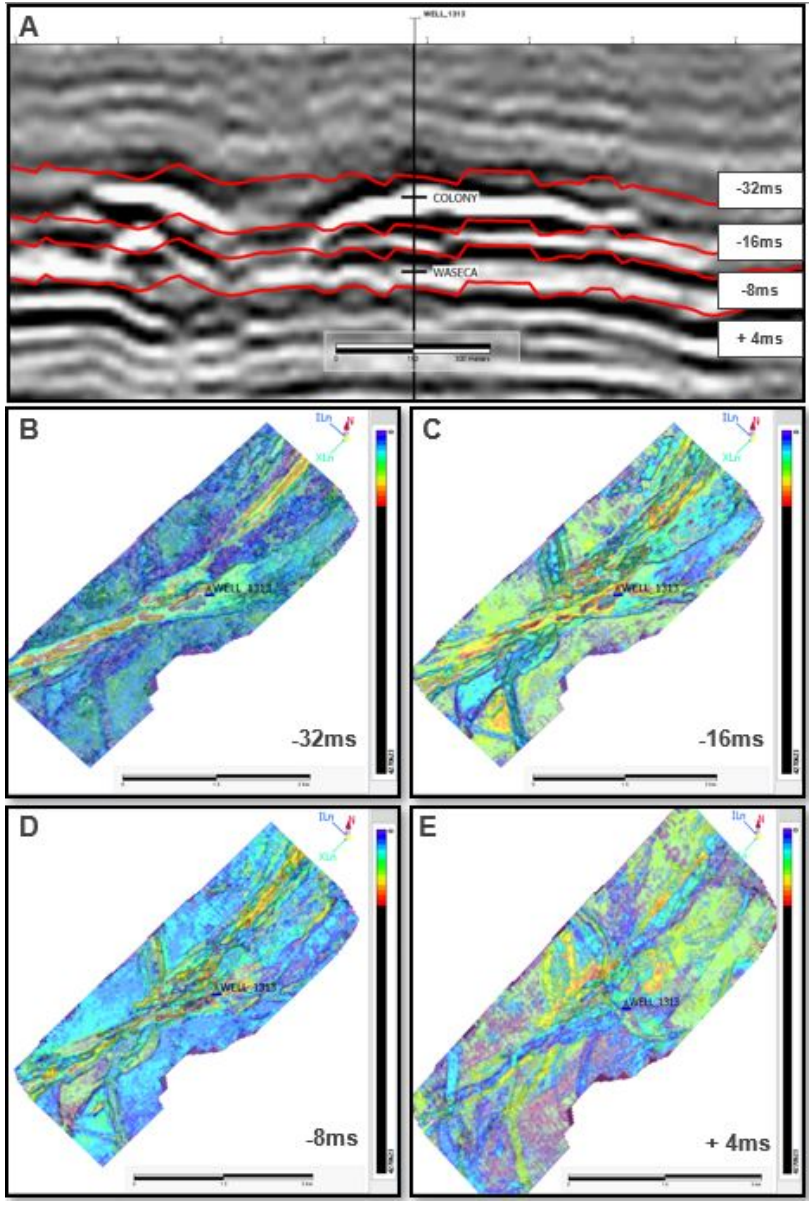

Figure 4: A) Seismic amplitude section and horizon slices extracted from Waseca horizon. Horizon slices at $-32 \mathrm{~ms}$ (B), -16ms (C), -8ms (D) and $4 m s$ (E), showing the variation of the channel system stratigraphy. The maps display the eigen coherence, spectral decomposition at $10 \mathrm{~Hz}$ and amplitude attributes blended by addition method.

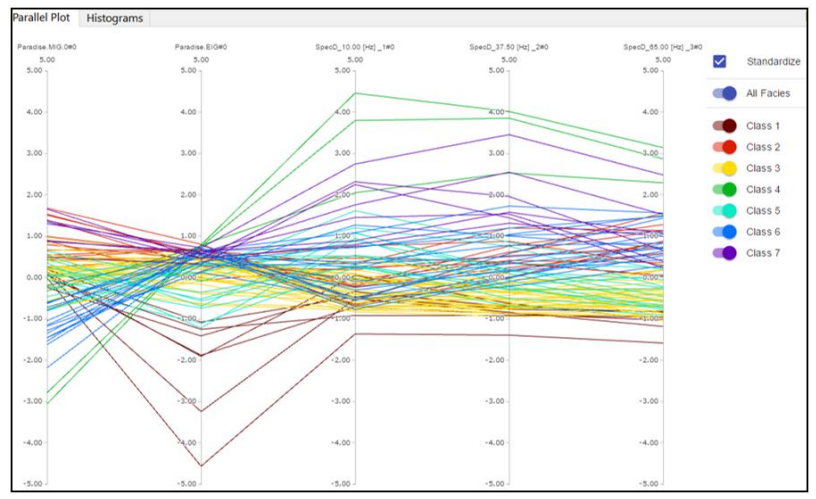

Figure 5: Parallel plot showing the neuron sets for each class in colors. The vertical lines correspond to the axis of the input attributes. A wide spread of the neurons indicates the attribute is useful for the prediction.

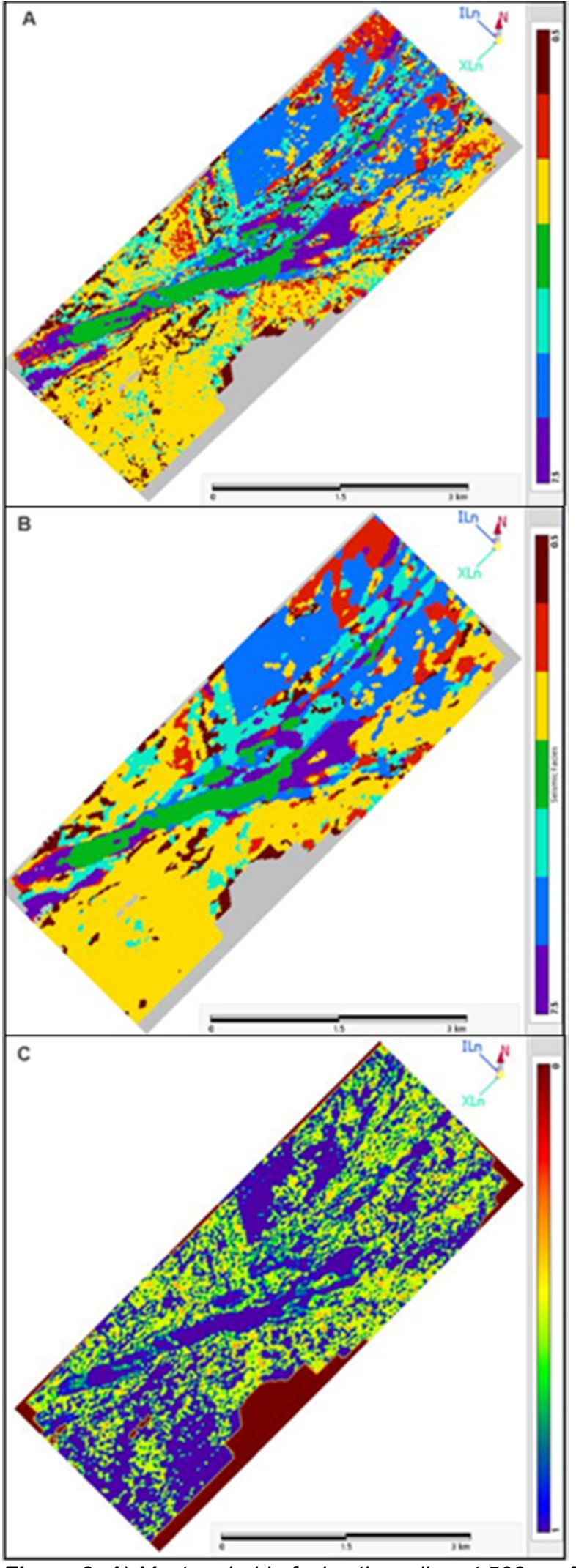

Figure 6: A) Most probable facies time slice at 506ms. B) Smoothed most probable facies time slice at $506 \mathrm{~ms}$. Green facies are interpreted as gas accumulations. C) 
Maximum facies probability time slice at $506 \mathrm{~ms}$. Blue values have the higher probability of occurrence.

The verification of $G R$ and density well logs in a production well match with the green facies interpreted as the gas accumulations (Figure 7). An automatic detection of this facies defined geobodies that can be helpful for better visualization and stratigraphy analysis, computation of gross rock volume or definition of new well locations (Figure 8).

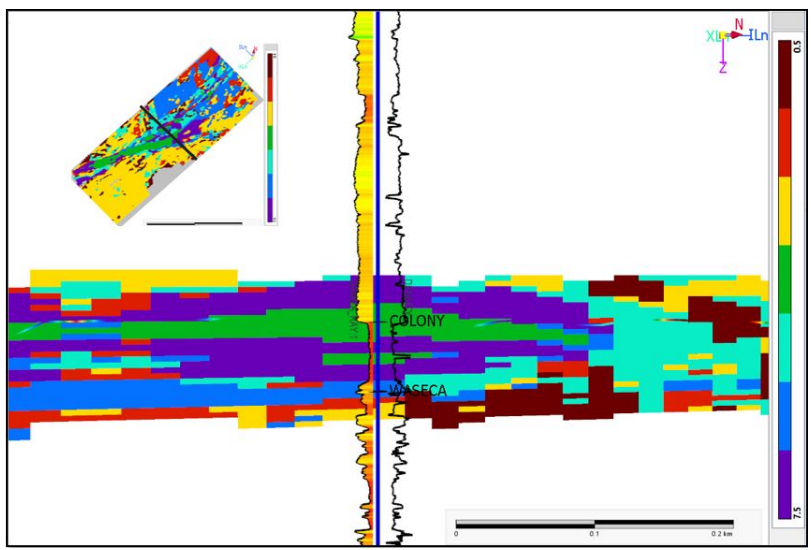

Figure 7: Cross-section at the producer well showing the most probable facies volume and the GR and density logs. The green facies interpreted as associated to the gas accumulation is consistent with the channel productive zone.
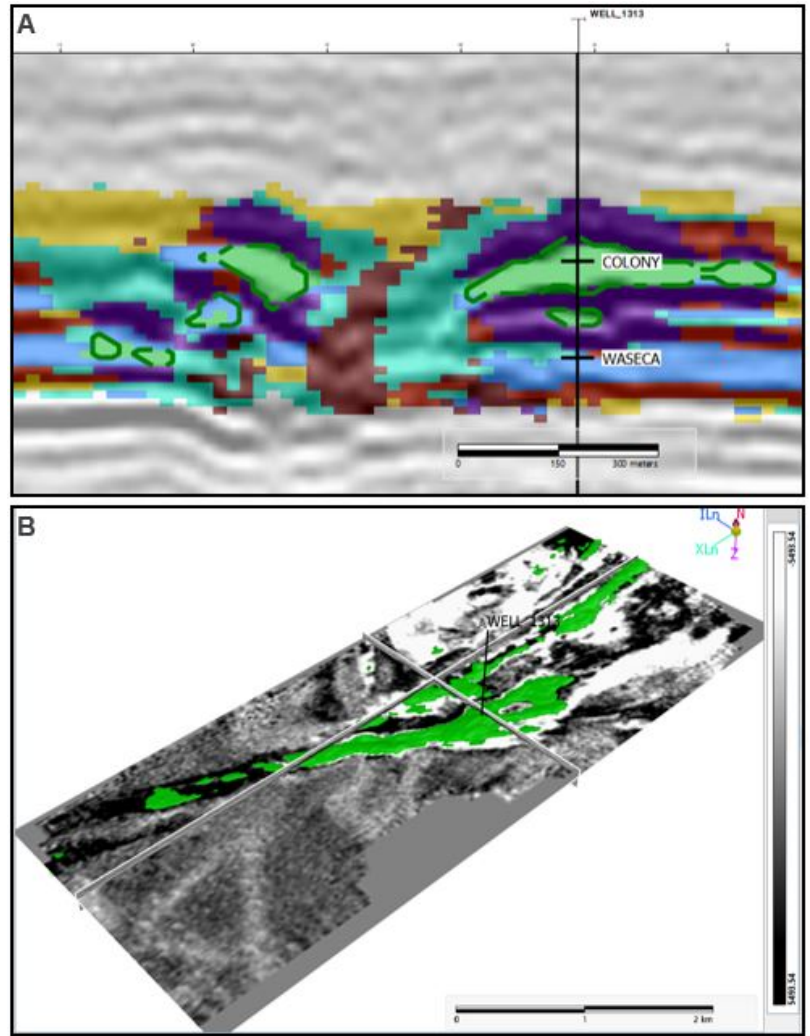

Figure 8: A) Seismic section with blending of amplitude and most probable facies at the location of the producer well. Notice the geobody wrap of the green facies that represents the gas accumulations. B) Detected geobodies displayed with the seismic amplitude, matching the brighter areas of the interpreted channel.

\section{Conclusions}

The selection of proper attributes and techniques for color blending, assist the interpreter to cleverly identify the features of interest in the stratigraphic analysis. They also help to decide what are the best inputs for facies classification. For our dataset, eigen coherence and spectral decomposition enable the clear identification of the channel system and the brighter anomalies related to gas accumulations. RGB was an effective technique for blending these attributes and obtain a better image.

The method chosen in this work for a non-supervised facies classification, the SGNN, has shown reliable results to identify gas accumulations based in key seismic attributes. Moreover, this workflow provides valuable information such as facies probabilities, which can feed uncertainty analysis associated to prospect identification and geological modeling.

This methodology can be used as a final stage to take advantage of several outputs from different seismic characterization workflows such as the inversion of amplitudes and structural analysis. 


\section{Acknowledgments}

The authors would like to thank Emerson for authorizing this publication.

\section{References}

Chopra, S. Marfurt, K. 2007. Seismic Attributes for Prospect Identification and Reservoir Characterization. SEG Geophysical Developments Series No 11.

Fritzke, B. 1995. A growing neural gas network learns topologies: Advances in neural information processing systems: MIT Press, 625-632

Hami-Eddine, K., Klein, P., Richard, L., de Ribet, B., Grout, M. 2015. A New Technique for Lithology and Fluid Content Prediction from Prestack Data: An Application to a Carbonate Reservoir. Interpretation 3 (1): SC19-32. doi:10.1190/INT-2014-0049.1

Hami-Eddine, K., Klein, P., de Ribet, B. 2015. Predicting reliability of AVA effects using neural networks. SEG Technical Program Expanded Abstracts: 567-570. https://doi.org/10.1190/segam2015-5908157.1

Henderson, J., Purves, S.J. and Leppard, C. 2007. Automated delineation of geological elements from 3D seismic data through analysis of multi-channel, volumetric spectral decomposition data. First Break, 25(3), 87-93.

Henderson, J., Purves, S., Fisher, G. and Leppard, C. 2008. Delineation of geological elements from RGB color blending of seismic attribute volumes. The Leading Edge, 27(3), 342-350.

Partyka, G., Gridley, J. and Lopez, J. 1999. Interpretational application of spectral decomposition in reservoir characterization: The Leading Edge, 353-360.

Schroeder. 2011. American Association of Petroleum Geologists. Seismic Facies Analysis. Available in https://archives.aapg.org/slide_resources/schroeder/11b/i ndex.cfm. Access in 10/26/2014.

Taner, M. T. 1992. Attributes Revisited - Rock Solid Images. Houston, Texas (Revised Sep. 2000), P. 3 - 27. 